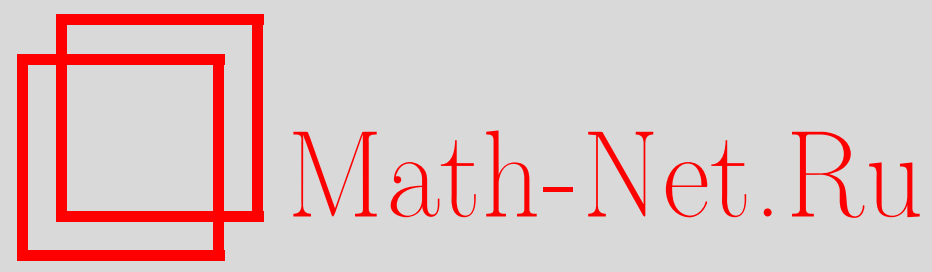

С. А. Степанов, $\mathrm{K}$ проблеме дискретного логарифма, Дискрет. матем., 2014, том 26, выпуск 1, 133-142

DOI: https://doi.org/10.4213/dm1273

Использование Общероссийского математического портала Math-Net.Ru подразумевает, что вы прочитали и согласны с пользовательским соглашением http://www . mathnet.ru/rus/agreement

Параметры загрузки:

IP: 18.209.158.208

26 апреля 2023 г., 18:15:48

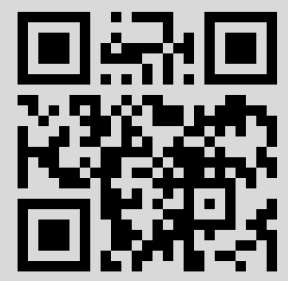




\title{
К проблеме дискретного логарифма
}

\author{
(c) 2014 г. $\quad$ C. А. Степанов ${ }^{1}$
}

\begin{abstract}
Пусть $\mathbb{F}_{q}$ - конечное поле характеристики $p$, состоящее из $q=p^{\nu}$ элементов, $g$ - примитивный элемент, $a \neq 0$ - произвольный элемент поля $\mathbb{F}_{q}$ и $x=\log _{g} a$ - дискретный логарифм элемента $a$ по основанию $g$. В работе рассматривается проблема дискретного логарифма в поле $\mathbb{F}_{q}, q \equiv 1(\bmod 4)$, и указывается полиномиальный относительно $q$ детерминистический алгоритм вычисления первых $k \leqslant c \log n$ знаков $x_{0}, x_{1}, \ldots, x_{k}, k<n$, бинарного представления $x=x_{0}+x_{1} 2+x_{2} 2^{2}+\cdots+x_{n} 2^{n}$ числа $x$.
\end{abstract}

Ключевые слова: конечное поле, примитивный элемент, дискретный логарифм, расширение конечной степени, норма элемента, полиномиальное уравнение диагонального вида.

\section{1. Введение}

Пусть $p$ - простое число $\mathbb{F}_{q}-$ конечное поле, состоящее из $q=p^{\nu}$ элементов. Мультипликативная группа $\mathbb{F}_{q}^{*}=\mathbb{F}_{q} \backslash\{0\}$ поля $\mathbb{F}_{q}$ является циклической группой порядка $q-1$. Порождающий элемент $g$ группы $\mathbb{F}_{q}^{*}$ называется примитивным элементом поля $\mathbb{F}_{q}$. Если $s-$ произвольное целое число, взаимно простое с $q-1$ и удовлетворяющее условию $1 \leqslant s \leqslant q-1$, то $g^{s}$ также является примитивным элементом поля $\mathbb{F}_{q}$. Отсюда следует, что поле $\mathbb{F}_{q}$ имеет в точности $\varphi(q-1)$ примитивных элементов, где $\varphi(n)$ - функция Эйлера. Каждый элемент $a \in \mathbb{F}_{q}^{*}$ единственным образом представим (см. [10, Глава 2], [22, Глава 1]) в виде

$$
g^{x}=a
$$

при некотором $x \in \mathbb{Z}, 0 \leqslant x \leqslant q-2$. Целое число $x$ в указанном представлении называется дискретным логарифмом (или же индексом) элемента а по основанию $g$ и обозначается через $\log a=\log _{g} a$ (или же через ind $a=\operatorname{ind}_{g} a$ ). Далее, проблема дискретного логарифма состоит в нахождении единственным образом определенного целого числа $x, 0 \leqslant x \leqslant q-2$, удовлетворяющего соотношению (1) при заданных $g$ и $a$.

$\mathrm{C}$ помощью повторного возведения в квадрат функция $y=g^{x}$ может быть весьма быстро вычислена для любого $x=0,1, \ldots, q-2$. В самом деле, если $x_{0}, x_{1}, x_{2}, \ldots, x_{n}$ - двоичные знаки числа $x$, т. е.

$$
x=x_{0}+x_{1} 2+x_{2} 2^{2}+\cdots+x_{n} 2^{n}, \quad n \leqslant[\log (q-2) / \log 2],
$$

\footnotetext{
${ }^{1}$ Место работы: Институт проблем передачи информации РАН e-mail: sa-stepanov@iitp.ru
} 
то мы имеем

$$
g^{x}=\prod_{\substack{i=0 \\ x_{i}=1}}^{n} g^{2^{i}} .
$$

Отсюда следует, что для вычисления $g^{x}$ требуется не более $2 n+1$ умножений в поле $\mathbb{F}_{q}$. Стало быть, если поле $\mathbb{F}_{q}$ задано, то время работы алгоритма, основанного на повторном возведении в квадрат, оценивается величиной $O\left(\log x \log ^{3} q\right)$ (детали см. в [8], стр. 22).

С другой стороны, нахождение обратной функции $x=\log _{g} y$ для больших значений $q$ рассматривается как весьма трудная задача (см. [2], [5], [6], [14], [21], [25]). Среди известных к настоящему времени быстрых алгоритмов вычисления дискретного логарифма в конечных полях можно выделить следующие четыре категории:

(i) Быстрые эвристические алгоритмы (LaMacchia, Odlyzko [9], McCurley [13]);

(ii) Субэкспоненциальные вероятностные алгоритмы, использующие случайные выборки параметров (Adleman [1], Coppersmith [3], Coppersmith, Odlyzko, Schroeppel [4], Hellman, Reyneri [7], Odlyzko [15], Pollard [17], Pomerance [18], Semaev [19]); в большинстве случаев эти алгоритмы работают эффективно лишь в случае, когда характеристика $p$ поля $\mathbb{F}_{q}$ мала;

(iii) Общие детерминистические алгоритмы, которые работают в произвольной конечной группе $G$ и вычисляют дискретный логарифм за $|G|^{1 / 2+\varepsilon}$ шагов (Massey [11], Shanks [20]);

(iv) Детерминистические алгоритмы, которые работают хорошо лишь когда все простые делители числа $q-1$ малы (McCurley [12], Pohlig, Hellman [16]).

В данной работе предлагается новый подход к проблеме дискретного логарифма и получен следующий результат.

Теорема. Пусть $p \geqslant 3$ - простое число , $\nu$ - положительное иелое число и $\mathbb{F}_{q}-$ конечное поле, состоящее из $q=p^{\nu}$ элементов. Если $q \equiv 1(\bmod 4)$, то существует детерминистический алгоритм, который для заданного поля $\mathbb{F}_{q}$, состоящего из $q=p^{\nu}$ элементов, заданного примитивного элемента $g$ поля $\mathbb{F}_{q}$ и произвольного ненулевого элемента $a \in \mathbb{F}_{q}$ определяет первые $k \leqslant n$ знаков $x_{0}, x_{1}, \ldots, x_{k-1}$ двоччного представления

$$
x=x_{0}+x_{1} 2+x_{2} 2^{2}+\cdots+x_{n} 2^{n}, \quad n \leqslant[\log (q-2) / \log 2],
$$

числа $x=\log _{g}$ а за $O\left(2^{2 k} \log ^{2} q\right)$ арифметических операций в поле $\mathbb{F}_{q^{2}}$.

Следствие. Пусть с и $\delta=\delta(\alpha)$ - положительные константы. Упомянутый выше алгоритм определяет $k \leqslant c \log n$ первых знаков бинарного представления

$$
x=x_{0}+x_{1} 2+x_{2} 2^{2}+\cdots+x_{n} 2^{n}
$$

числа $x=\log _{g}$ а за $O\left(\log ^{\delta} q\right)$ операций в поле $\mathbb{F}_{q^{2^{k}}}$. 


\section{2. Описание алгоритма}

Для дальнейших рассмотрений нам удобно положить

$$
\begin{gathered}
g=g_{0}, \quad a=a_{0}, \quad x=x_{0}+x_{1} 2+x_{2} 2^{2}+\cdots+x_{n} 2^{n}, \\
0 \leqslant x_{0}+x_{1} 2+x_{2} 2^{2}+\cdots+x_{n} 2^{n} \leqslant q-2,
\end{gathered}
$$

где $x_{i} \in\{0,1\}, 0 \leqslant i \leqslant n$, и затем переписать соотношение (1) в виде

$$
g_{0}^{x_{0}+x_{1} 2+x_{2} 2^{2}+\cdots+x_{n} 2^{n}}=a_{0} .
$$

Возводя обе части последнего соотношения в степень $(q-1) / 2$ и учитывая, что

$$
g_{0}^{\frac{q-1}{2}\left(x_{1} 2+x_{2} 2^{2}+\cdots+x_{n} 2^{n}\right)}=g_{0}^{(q-1)\left(x_{1}+2 x_{2}+\cdots+x_{n} 2^{n-1}\right)}=1
$$

в поле $\mathbb{F}_{q}$, мы приходим к равенству

$$
g_{0}^{x_{0} \frac{q-1}{2}}=a_{0}^{\frac{q-1}{2}} .
$$

Затем, учитывая, что $g_{0}^{(q-1) / 2}=-1$, получаем соотношение

$$
(-1)^{x_{0}}=a_{0}^{\frac{q-1}{2}},
$$

которое дает возможность единственным образом определить $x_{0}$ :

$$
x_{0}= \begin{cases}0, & \text { если } a_{0}-\text { квадрат в } \mathbb{F}_{q}, \\ 1 & \text { в противном случае. }\end{cases}
$$

После этого уравнение (2) может быть переписано в виде

$$
g_{0}^{2\left(x_{1}+x_{2} 2+\cdots+x_{n} 2^{n-1}\right)}=\tilde{a}_{0},
$$

где $\tilde{a}_{0}=a_{0} g^{-x_{0}}$.

Обозначим через $g_{1}$ такой примитивный элемент квадратичного расширения $\mathbb{F}_{q^{2}}$ поля $\mathbb{F}_{q}$, что

$$
\operatorname{Norm}_{\mathbb{F}_{q^{2}} / \mathbb{F}_{q}} g_{1}=g_{1}^{1+q}=g_{0},
$$

а через $a_{1}=\alpha_{1}^{2}$ обозначим такой элемент поля $\mathbb{F}_{q^{2}}$, что

$$
\operatorname{Norm}_{\mathbb{F}_{q^{2}} / \mathbb{F}_{q}} a_{1}=a_{1}^{1+q}=\tilde{a}_{0} .
$$

Затем рассмотрим в поле $\mathbb{F}_{q^{2}}$ уравнение

$$
g_{1}^{2\left(x_{1}+x_{2} 2+\cdots+x_{n} 2^{n-1}\right)}=a_{1},
$$

которое получается поднятием уравнения (3) из $\mathbb{F}_{q}$ в поле $\mathbb{F}_{q^{2}}$. Возводя обе части последнего уравнения в степень $\left(q^{2}-1\right) / 2^{2}$ и принимая во внимание, что

$$
g_{1}^{\frac{q^{2}-1}{2}}=-1
$$


мы приходим к равенству

$$
(-1)^{x_{1}}=a_{1}^{\frac{\left(q^{2}-1\right)}{2^{2}}}=\alpha_{1}^{\frac{q^{2}-1}{2}},
$$

которое дает

$$
x_{1}= \begin{cases}0, & \text { если } \alpha-\text { квадрат в } \mathbb{F}_{q^{2}}, \\ 1 & \text { в противном случае. }\end{cases}
$$

Покажем теперь, что указанное выше значение $x_{1}$ не зависит от конкретного выбора определенного выше поднятия. Зафиксируем элемент $g_{1}$ и вместо уравнения (4) рассмотрим уравнение

$$
g_{1}^{2\left(x_{1}+x_{2} 2+\cdots+x_{n} 2^{n-1}\right)}=b_{1}
$$

с такой правой частью $b_{1}=a_{1} g_{1}^{(q-1) t_{1}} \in \mathbb{F}_{q^{2}}, t_{1} \in \mathbb{Z}$, что

$$
\operatorname{Norm}_{\mathbb{F}_{q^{2}} / \mathbb{F}_{q}} b_{1}=b_{1}^{1+q}=\tilde{a_{0}} .
$$

Возводя обе части последнего уравнения в степень $\left(q^{2}-1\right) / 2^{2}$ и принимая затем во внимание, что

$$
g_{1}^{\frac{q^{2}-1}{2}}=-1, \quad q-1 \equiv 0\left(\bmod 2^{2}\right),
$$

мы приходим к соотношению

$$
(-1)^{x_{1}}=a_{1}^{\frac{q^{2}-1}{2^{2}}}\left(g_{1}^{\frac{(q-1) t_{1}}{2^{2}}}\right)^{\left(q^{2}-1\right)}=\alpha_{1}^{\frac{q^{2}-1}{2}},
$$

которое задает в точности то же самое, что и прежде, значение для $x_{1} \in\{0,1\}$. В результате мы можем предположить без уменьшения общности, что $t_{1}=0$, и затем переписать уравнение (4) в виде

$$
g_{1}^{2^{2}\left(x_{2}+x_{3} 2+\cdots+x_{n} 2^{n-2}\right)}=\tilde{a}_{1},
$$

где $\tilde{a}_{1}=a_{1} g_{1}^{-2 x_{1}}$.

На втором шаге выберем некоторый примитивный элемент $g_{2}$ квадратичного расширения $\mathbb{F}_{q^{2}}$ поля $\mathbb{F}_{q^{2}}$, удовлетворяющий условию

$$
\operatorname{Norm}_{\mathbb{F}_{q^{2}} / \mathbb{F}_{q^{2}}} g_{2}=g_{2}^{1+q^{2}}=g_{1},
$$

и обозначим через $a_{2}=\alpha_{2}^{2^{2}}$ такой элемент поля $\mathbb{F}_{q^{2}}$, что

$$
\operatorname{Norm}_{\mathbb{F}_{q^{2}} / \mathbb{F}_{q^{2}}} a_{2}=a_{2}^{1+q^{2}}=\tilde{a}_{1} .
$$

Далее, рассмотрим в поле $\mathbb{F}_{q^{2}}$ соотношение

$$
g_{2}^{2^{2}\left(x_{2}+x_{3} 2+\cdots+x_{n} 2^{n-2}\right)}=a_{2},
$$

получаемое поднятием уравнения (5) из поля $\mathbb{F}_{q^{2}}$ в поле $\mathbb{F}_{q^{2}}$. Возводя обе части последнего уравнения в степень $\left(q^{2^{2}}-1\right) / 2^{3}$ и принимая во внимание, что

$$
g_{2}^{\frac{q^{2^{2}}-1}{2}}=-1
$$


получаем равенство

$$
(-1)^{x_{2}}=a_{2}^{\frac{q^{2^{2}}-1}{2^{3}}}=\alpha_{2}^{\frac{q^{2^{2}}-1}{2}},
$$

которое приводит к соотношению

$$
x_{2}= \begin{cases}1, & \text { если } \alpha_{2}-\text { квадрат в } \mathbb{F}_{q^{2}}, \\ 0 & \text { в противном случае. }\end{cases}
$$

Теперь мы покажем снова, что значение величины $x_{2}$ не зависит от конкретного выбора определенного выше поднятия. Зафиксируем примитивный элемент $g_{2}$ поля $\mathbb{F}_{q^{2}}$ и рассмотрим уравнение

$$
g_{2}^{2^{2}\left(x_{2}+x_{3} 2+\cdots+x_{n} 2^{n-2}\right)}=b_{2}
$$

с такой правой частью $b_{2}=a_{2} g_{2}^{\left(q^{2}-1\right) t_{2}} \in \mathbb{F}_{q^{2^{2}}}, t_{2} \in \mathbb{Z}$, что

$$
\operatorname{Norm}_{\mathbb{F}_{q^{2}} / \mathbb{F}_{q^{2}}} b_{2}=b_{2}^{1+q^{2}}=\tilde{a}_{1} \text {. }
$$

Возводя обе части последнего уравнения в степень $\left(q^{2^{2}}-1\right) / 2^{3}$ и принимая во внимание, что

$$
g_{2}^{\frac{q^{2^{2}}-1}{2}}=-1, \quad q^{2^{2}}-1 \equiv 0\left(\bmod 2^{3}\right),
$$

приходим к соотношению

$$
(-1)^{x_{2}}=a_{2}^{\frac{q^{2^{2}}-1}{2^{3}}}\left(g_{2}^{\frac{\left(q^{2}-1\right) t_{2}}{2^{3}}}\right)^{\left(q^{2^{2}}-1\right)}=\alpha_{2}^{\frac{q^{2^{2}}-1}{2}},
$$

которое единственным образом определяет в точности то же самое значение $x_{2} \in$ $\{0,1\}$, что и найденное ранее. Теперь мы снова можем считать без уменьшения общности, что $t_{2}=0$, а затем переписать уравнение (6) в виде

$$
g_{2}^{2^{3}\left(x_{3}+x_{4} 2+\cdots+x_{n} 2^{n-3}\right)}=\tilde{a}_{2}
$$

где $\tilde{a}_{2}=a_{2} g_{2}^{-2^{2} x_{2}}$.

Повторяя описанный выше процесс $i$ раз, где $0 \leqslant i \leqslant n-1$, мы приходим к соотношению

$$
g_{i}^{2^{i+1}\left(x_{i+1}+x_{i+2} 2+\cdots+x_{n} 2^{n-i-1}\right)}=\tilde{a}_{i},
$$

где $g_{i}$ - некоторый примитивный элемент поля $\mathbb{F}_{q^{2^{i}}}$ и $\tilde{a}_{i}-$ некоторый элемент этого поля, полученный из исходного элемента $a \in \mathbb{F}_{q}$ на предыдущем шаге. Пусть $g_{i+1}-$ некоторый примитивный элемент квадратичного расширения $\mathbb{F}_{q^{2^{i+1}}}$ поля $\mathbb{F}_{q^{2}}$ удовлетворяющий условию

$$
\operatorname{Norm}_{\mathbb{F}_{q^{2}}{ }^{i+1} / \mathbb{F}_{q^{2^{i}}}} g_{i+1}=g_{i+1}^{1+q^{2^{i}}}=g_{i},
$$

a $a_{i+1}=\alpha_{i+1}^{2^{i+1}}-$ некоторый элемент поля $\mathbb{F}_{q^{2^{i+1}}}$ удовлетворяющий условию

$$
\operatorname{Norm}_{\mathbb{F}_{q^{2^{i+1}}} / \mathbb{F}_{q^{2^{i}}}} a_{i+1}=a_{i+1}^{1+q^{2^{i}}}=\tilde{a}_{i} .
$$


Рассмотрим в поле $\mathbb{F}_{q^{2^{i+1}}}$ уравнение

$$
g_{i+1}^{2^{i+1}\left(x_{i+1}+x_{i+2} 2+\cdots+x_{n} 2^{n-i-1}\right)}=a_{i+1},
$$

получаемое поднятием уравнения $(7)$ из поля $\mathbb{F}_{q^{2^{i}}}$ в расширенное поле $\mathbb{F}_{q^{2}}{ }^{i+1}$. Возводя обе части последнего уравнения в степень $\left(q^{2^{i+1}}-1\right) / 2^{i+2}$ и принимая во внимание, что

$$
g_{i+1}^{\frac{\left(q^{2^{i+1}}-1\right)}{2}}=-1
$$

мы приходим к уравнению

$$
(-1)^{x_{i+1}}=a_{i+1}^{\frac{q^{2^{i+1}}-1}{2^{i+2}}}=\alpha_{i+1}^{\frac{q^{2^{i+1}}-1}{{ }^{2}}},
$$

которое дает

$$
x_{i+1}=\left\{\begin{array}{l}
1, \quad \text { если } \alpha_{i+1} \text { квадрат в } \mathbb{F}_{q^{2}}{ }^{i+1}, \\
0 \quad \text { в противном случае. }
\end{array}\right.
$$

Теперь мы покажем снова, что значение величины $x_{i+1}$ не зависит от конкретного выбора указанного выше поднятия уравнения (7). Зафиксируем примитивный элемент $g_{i+1}$ поля $\mathbb{F}_{q^{2^{i+1}}}$ и рассмотрим уравнение

$$
g_{i+1}^{2^{i+1}\left(x_{i+1}+x_{i+2} 2+\cdots+x_{n}^{n-i-1}\right)}=b_{i+1}
$$

с некоторым другим членом $b_{i+1}=a_{i+1} g_{i+1}^{\left(q^{2^{i+1}}-1\right) t_{i+1}} \in \mathbb{F}_{q^{2^{i+1}}}, t_{i+1} \in \mathbb{Z}$, в правой части, удовлетворяющим соотношению

$$
\operatorname{Norm}_{\mathbb{F}_{q^{2^{i+1}}} / \mathbb{F}_{q^{2^{i}}}} b_{i+1}=b_{i+1}^{1+q^{2^{i}}}=\tilde{a}_{i} .
$$

Возводя обе части последнего уравнения в степень $\left(q^{2^{i+1}}-1\right) / 2^{i+2}$ и принимая во внимание, что

$$
g_{i+1}^{\frac{\left(q^{2^{i+1}}-1\right)}{2}}=-1, \quad q^{2^{i+1}}-1 \equiv 0\left(\bmod 2^{i+2}\right),
$$

мы приходим к соотношению

$$
(-1)^{x_{i+1}}=a_{i+1}^{\frac{q^{2^{i+1}}-1}{2^{i+2}}}\left(g_{i+1}^{\frac{\left(q^{2^{i+1}}-1\right) t_{i+1}}{2^{i+2}}}\right)^{\left(q^{2^{i+1}}-1\right)}=\alpha_{i+1}^{\frac{q^{2^{i+1}}-1}{2}},
$$

которое единственным образом определяет то же самое значение $x_{i+1} \in\{0,1\}$, что и указанное ранее. В результате, мы можем предположить без уменьшения общности, что $t_{i+1}=0$, и затем переписать соотношение (8) в виде

$$
g_{i+1}^{2^{i+2}\left(x_{i+2}+x_{i+3} 2+\cdots+x_{n} 2^{n-i-2}\right)}=\tilde{a}_{i+1},
$$

где $\tilde{a}_{i+1}=a_{i+1} g_{i+1}^{-2^{i+1} x_{i+1}}$.

На заключительном $(n-1)$-м шаге мы приходим к соотношению

$$
g_{n-1}^{2^{n} x_{n}}=\tilde{a}_{n-1}
$$


где $g_{n-1}-$ некоторый примитивный элемент поля $\mathbb{F}_{q^{2}{ }^{n-1}}$ и $\tilde{a}_{n-1}-$ некоторый элемент того же поля. Рассмотрим примитивный элемент $g_{n}$ поля $\mathbb{F}_{q^{2}}$, удовлетворяющий соотношению

$$
\operatorname{Norm}_{\mathbb{F}_{q^{2}} / \mathbb{F}_{q^{2}}} g_{n}=g_{n}^{1+q^{2^{n-1}}}=g_{n-1},
$$

и обозначим через $a_{n}=\alpha_{n}^{2^{n}}$ некоторый элемент поля $\mathbb{F}_{q^{2}}$, удовлетворяющий соотношению

$$
\operatorname{Norm}_{\mathbb{q}_{q^{2}} / \mathbb{F}_{q^{2}-1}} a_{n}=a_{n}^{1+q^{2^{n-1}}}=\tilde{a}_{n-1} .
$$

Далее, рассмотрим уравнение

$$
g_{n}^{2^{n} x_{n}}=a_{n},
$$

получаемое поднятием соотношения (9) из поля $\mathbb{F}_{q^{2^{n-1}}}$ в поле $\mathbb{F}_{q^{2 n}}$, и заметим, что

$$
q^{2^{n}}-1 \equiv 0\left(\bmod 2^{n+1}\right) .
$$

Возводя обе части равенства $(10)$ в степень $\left(q^{2^{n}}-1\right) / 2^{n+1}$ и принимая во внимание, что

$$
g_{n}^{\frac{q^{2^{n}}-1}{2}}=-1
$$

мы приходим к соотношению

$$
(-1)^{x_{n}}=a_{n^{\frac{q^{n}}{2^{n+1}}}}=\alpha_{n}^{\frac{q^{2^{n}}-1}{2}},
$$

которое показывает, что

$$
x_{n}= \begin{cases}1, & \text { если } \alpha_{n} \text { квадрат в } \mathbb{F}_{q^{2}}, \\ 0 & \text { в противном случае. }\end{cases}
$$

Наконец, покажем тем же образом, как и ранее, что полученное нами значение величины $x_{n}$ не зависит от конкретного выбора указанного выше поднятия. В самом деле, зафиксируем $g_{n}$ и рассмотрим уравнение

$$
g_{n}^{2^{n} x_{n}}=b_{n}
$$

с некоторым другим членом $b_{n}=a_{n} g_{n}^{\left(q^{2^{n}}-1\right) t_{n}}, t_{n} \in \mathbb{Z}$, в правой части, таким, что

$$
\operatorname{Norm}_{\mathbb{F}_{q^{2}} / \mathbb{F}_{q^{2^{n-1}}}} b_{n}=b_{n}^{1+q^{2^{n-1}}}=\tilde{a}_{n-1} \text {. }
$$

Возводя теперь обе части последнего уравнения в степень $\left(q^{2^{n}}-1\right) / 2^{n+1}$ и принимая во внимание, что

$$
g_{n}^{\frac{\left(2^{2^{n}}-1\right)}{2}}=-1, \quad q^{2^{n}}-1 \equiv 0\left(\bmod 2^{n+1}\right),
$$

приходим к соотношению

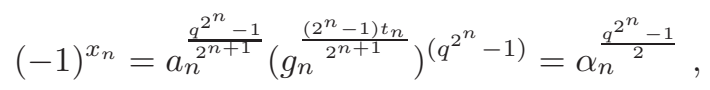

которое определяет то же самое значение величины $x_{n} \in\{0,1\}$, что и указанное ранее. Предположив снова, что $t_{n}=0$, и поделив затем обе части равенства (10) на $g_{n}^{2^{n}} x_{n}$, мы приходим к соотношению

$$
1=a_{n} g_{n}^{-2^{n} x_{n}},
$$

которое завершает рассматриваемый процесс. 


\section{3. Вычислительная сложность алгоритма}

Оценим время работы первых $k \leqslant n$ шагов описанного выше алгоритма. Наши аргументы существенно опираются на следующий результат ([23], Теорема 7.1).

Предложение. Существует детерминистический алгоритм, который для заданного конечного поля $\mathbb{F}_{q}$, состоящего из q элементов, положительного иелого $\mathrm{m}$, делящего $q-1$, и ненулевых элементов $a_{1}, \ldots, a_{m}, b \in \mathbb{F}_{q}$, находит такие элементы $x_{1}, \ldots, x_{m} \in \mathbb{F}_{q}$, чmo

$$
\sum_{i=1}^{m} a_{i} x_{i}^{m}=b,
$$

или решает, что таких элементов не существует, используя

$$
\tilde{O}\left(m^{3} \log q+m^{2} \log ^{2} q\right)
$$

операчий в поле $\mathbb{F}_{q}$.

Следует отметить, что граница сложности $\tilde{O}\left(m^{3} \log q+m^{2} \log ^{2} q\right)$, указанная в Предложении, является «мягкой» относительно $m$; это означает, что логарифмические множители вида $\log ^{l} m$ игнорируются (точное определение см. в [24, раздел 25.7]).

Напомним, что каждый из $k \leqslant n$ шагов нашего алгоритма состоит из некоторого подходящего поднятия обеих частей уравнения

$$
g_{i-1}^{2^{i}\left(x_{i}+x_{i+1} 2+\cdots+x_{n} 2^{n-i}\right)}=\tilde{a}_{i}
$$

из поля $\mathbb{F}_{q^{2^{i-1}}}$ в поле $\mathbb{F}_{q^{2^{i}}}$. Напомним также, что для того, чтобы поднять левую часть уравнения (11), мы должны указать такой примитивный элемент $g_{i}$ поля $\mathbb{F}_{q^{2}}$, что

$$
\operatorname{Norm}_{\mathbb{F}_{q^{2^{i}}} / \mathbb{F}_{q^{2}}} g_{i}=g_{i-1} \text {. }
$$

Пусть $\left\{1, \omega_{i}\right\}$ - некоторый базис поля $\mathbb{F}_{q^{2^{i}}}$ над $\mathbb{F}^{q^{2^{i-1}}}$ и

$$
g_{i}=u_{i 1}+u_{i 2} \omega_{i}, \quad u_{i 1}, u_{i 2} \in \mathbb{F}_{q^{2^{i-1}}} .
$$

Мы имеем

$$
\operatorname{Norm}_{\mathbb{F}_{q^{2^{i}}} / \mathbb{F}_{q^{2^{i-1}}}} g_{i}=\left(u_{i 1}+u_{i 2} \omega_{i}\right)\left(u_{i 1}+u_{i 2} \omega_{i}^{q^{2^{i-1}}}\right)=u_{i 1}^{2}+a_{i} u_{i 2}+b_{i} u_{i 2}^{2},
$$

где $a_{i}, b_{i} \in \mathbb{F}_{q^{2 i-1}}$. Записывая теперь последнее соотношение в виде

$$
\operatorname{Norm}_{\mathbb{F}_{q^{2^{i}}} / \mathbb{F}_{q^{2^{i-1}}}} g_{i}=v_{i 1}^{2}+c_{i} v_{i 2}^{2},
$$

где

$$
\begin{gathered}
v_{i 1}=u_{i 1}+\frac{\omega_{i}+\omega_{i}^{q^{2^{i-1}}}}{2} u_{i 2}, \quad v_{i 2}=u_{i 2}, \\
c_{i}=\left(\omega_{i} \omega_{i}^{q^{i-1}}-\frac{\left(\omega_{i}+\omega_{i}^{q^{2^{i-1}}}\right)^{2}}{4}\right) \neq 0,
\end{gathered}
$$


и принимая во внимание сформулированное выше Предложение, мы находим, что вычисление элемента $g_{i}$ требует $O\left(2^{i} \log ^{2} q\right)$ арифметических операций в поле $\mathbb{F}_{q^{2^{i}}}$. Аналогично, построение такого элемента $a_{i} \in \mathbb{F}_{q^{2^{i}}}$, что

$$
\operatorname{Norm}_{\mathbb{F}_{q^{2}} / \mathbb{F}_{q^{2}}} a_{i}=\tilde{a}_{i-1}
$$

требует $O\left(2^{i} \log ^{2} q\right)$ операций в поле $\mathbb{F}_{q^{2}}$.

Таким образом, мы можем вычислить $k$ первых бинарных знаков $x_{0}, x_{1}, \ldots, x_{k-1}$ двоичного представления

$$
x=x_{0}+x_{1} 2+x_{2} 2^{2}+\cdots+x_{n} 2^{n}
$$

числа $x=\log _{g} a$, используя $O\left(2^{2 k} \log ^{2} q\right)$ арифметических операций в конечном поле $\mathbb{F}_{q^{2}}$.

\section{Список литературы}

1. Adleman L.M., A sub-exponential algorithm for the discrete logarithm problem with applications. Proc. 20-th Annual IEEEE Symp. on the Found. Comp. Sci. (1979) 55-60.

2. Diffie W., Hellman M., New directions in cryptography. IEEE Trans. Inf. Theory (1976) 22, 644-654.

3. Coppersmith D., Fast evaluation of discrete logarithms in fields of characteristic 2. IEEE Trans. Inf. Theory (1984) 30, 587-594.

4. Coppersmith D., Odlyzko A.M., Schroeppel R., Discrete logarithms in GF(p). Algoritmica (1986) 1, 1-15.

5. ElGamal T., A public key cryptosystem and a signature scheme based on discrete logarithms. IEEE Trans. Inf. Theory (1985) 31, №4, 469-472.

6. ElGamal T., Computing logarithms over finite fields, Lect. Notes in Comp. Sci. (1986) 218, 396-402.

7. Hellman M.E., Reyneri J.M., Fast computation of discrete logarithms in $G F(q)$. Proc. of Cripto' 82 (1983) 3-13.

8. Koblitz N., A Course in Number Theory and Cryptography. Springer-Verlag, New York, 1987.

9. LaMacchia B.A., Odlyzko A.M., Computation of discrete logarithms in prime fields. Designs, Codes and Cryptography (1991) 1, №1, 47-62.

10. Lidl R., Niederreiter G.L., Finite Fields. Addison-Wesley, Reading, MA, 1983.

11. Massey J.L., Logarithms in finite cyclic groups - cryptographic issues. Proc. 4-th Benelux Symp. on Inf. Theory (1983) 17-25.

12. McCurley K.S., The discrete logarithm problem. Proc. Symp. in Appl. Math. (1990) 42, 49-74.

13. McCurley K.S., Cryptographic key distribution and computation in class groups. Proc. of the NATO Advanced Study Inst. on Number Theory and Appl. Kluwer, Boston (1989) 459-479.

14. Menezes A., Okamoto T., Vanstone S., Reducing elliptic curve logarithms to logarithms in a finite field. Proc. 22-th Annual ACM Symp. on Theory of Comp. (1991) 80-89.

15. Odlyzko A.M., Discrete logarithms in finite fields and their cryptographic significance. Proc. of Eurocrypt 84 (1985) 224-314. 
16. Pohlig S., Hellman M., An improved algorithm for computing logarithms over $G F(p)$ and its cryptographic significance. IEEE Trans. Inf. Theory (1978) 24, №1, 106-110.

17. Pollard J.M., Monte Carlo methods for index computation mod p. Math. Comp. (1978) 32, 918-924.

18. Pomerance C., Fast, rigorous factorization and discrete logarithm algorithms. Discrete Algorithms and Complexity (1987) 119-143.

19. Semaev I.A., Computation of discrete logarithms in an arbitrary finite field. Discrete Math. Appl. (1995) 5, №2, 107-116.

20. Shanks D., Class number, a theory of factorization and genera. Proc. Symp. in Pure Math. (1971) 415-440.

21. Shparlinski I.E., Computational and algorithmic problems in finite fields (1992).

22. Stepanov S.A., Arithmetic of algebraic curves. Plenum, New York - London, 1994.

23. Van de Woestijne C., Deterministic equation solving over finite fields. ISSAC'05 (2005).

24. Von zur Gathen J., Gerhard J., Modern computer algebra (second, ed.). Cambridge University Press, 2003.

25. Washington L., Elliptic curves: number theory and cryptography. Chapman \& Hall / CRC Press, 2003.

Статья поступила 02.07.2013. 